\title{
Quasi-Periodic Orbits of the Restricted Three-Body Problem Made Easy
}

\author{
Egemen Kolemen*, N. Jeremy Kasdin ${ }^{\dagger}$ and Pini Gurfil** \\ *Mechanical and Aerospace Engineering, Princeton, NJ 08544, ekolemen@ princeton.edu \\ ${ }^{\dagger}$ Mechanical and Aerospace Engineering, Princeton, NJ 08544 \\ ${ }^{* *}$ Faculty of Aerospace Engineering, Technion - Israel Institute of Technology, Technion, Haifa 32000, Israel
}

\begin{abstract}
.
A new fully numerical method is presented which employs multiple Poincaré sections to find quasi-periodic orbits. The main advantages of this method are the small overhead cost of programming and very fast execution times, robust behavior near chaotic regions that leads to full convergence for given family of quasi-periodic orbits and the minimal memory required to store these orbits. This method reduces the calculation of the search for the two-dimensional invariant torus to a search for the closed orbits, which are the intersection of the invariant torus with the Poincaré sections. Truncated Fourier series are employed to represent these closed orbits. The flow of the differential equation on the invariant torus is reduced to maps between the consecutive Poincaré maps. A Newton iteration scheme makes use of the invariancy of the circles of the maps on these Poincaré sections in order to find the Fourier coefficient that define the circles to any given accuracy. A continuation procedure that uses the incremental behavior of the Fourier coefficients between close quasi-periodic orbits is utilized to extend the results from a single orbit to a family of orbits. Quasi-Halo and Lissajous families of the Sun-Earth Restricted Three-Body Problem (RTBP) around the L1 and L2 libration points are obtained via this method. Results are compared with the existing literature.
\end{abstract}

Keywords: Quasi-Periodic Orbit, Quasi-Halo, Lissajous Orbit, Restricted Three-Body Problem, Poincaré Section PACS: $95.55 . \mathrm{Pe}$

\section{INTRODUCTION}

The Libration points of the Earth-Moon and Sun-Earth have many advantageous properties that make them desirable for space missions, such as a stable thermal environment for infrared space telescopes and continuous coverage of the backside of the Moon for communication with future Moon bases. These properties have enabled existing and planned missions, such as the James Webb Space Telescope (JWST) and Wilkinson Microwave Anisotropy Probe (WMAP), among others. With the many future missions planned for these regions, the need for efficient approaches for trajectory design is apparent.

Since the mass of the satellite is so small relative to the larger bodies, it is sufficient to consider the Restricted Three Body Problem (RTBP) for the design of trajectories [1]. In order to minimize station-keeping, periodic orbits are of great interest. Using various methods, Farquhar [2, 3], Hénon [4], Breakwell [5], Howell [6] and others successfully obtained and studied the three types of periodic orbits around the libration points, namely halo, vertical and horizontal Lyapunov orbits.

Recently, a proposal has been put forward to place a new generation of formation flying missions, such as TPFI, Con-X, or the New World Discoverer, in orbits about the Sun-Earth L2 libration point. One way of reducing fuel consumption is to place all of the spacecraft in the constellation on a quasi-periodic orbit, thus maintaining a maximum separation. Because of the large separation distances, however, linear analysis and control is not possible.

In this paper, we only briefly review the literature for the RTBP; for background on general methods to find invariant tori, see [7]. There have been, though, a number of studies targeted at finding the quasi-periodic orbits around the libration points. Howell et al. used an ad hoc shooting method to find the Lissajous orbits [8]. However, the period of the orbit cannot be specified and there is no apparent way of continuation. Barden applied this method to find quasi-halos [9, 10]. Jorba et al. used the normal form method to find the center manifold around libration methods $[11,12]$. This method can be used to find the quasi-periodic orbits but the periods of the orbits cannot be specified or be based on series expansions. This method cannot achieve high accuracy. Gómez et al. used semi-analytic methods (Lindstedt-Poincaré procedure) to find the quasi-periodic orbits around libration points. A significant drawback of this method is that it has a low radius of convergence, and that a new code must be written for each orbit family. Coding this 
semi-analytic algorithm is time-consuming and is thus difficult to implement [13]. Gómez and Mondelo used a refined Fourier analysis to find the full families but this method is very slow and it is implemented on a cluster of parallel computers $[14,15]$. In this paper we present a fast, efficient, and easy to implement approach for finding quasi-periodic trajectories around Halo or Lyapunov L2 orbits.

We structure the paper as follows: First, an overview is given of the periodic and quasi-periodic orbits around the libration points. Next, the numerical procedure that finds the quasi-periodic orbits using a single Poincaré section is explained. The procedure is then generalized to multiple sections. The continuation procedure for extending the results to a family of periodic orbits is discussed, as are the different implementations of the method. Finally, the complete Quasi-Halo and Lissajous families are obtained and a comparison with the literature is provided for the fixed period and energy.

For the sake of conciseness, this paper focuses on the phase space around Sun-Earth L2 libration point. The results can be extended trivially to other collinear libration points around Sun-Earth and Earth-Moon system.

\section{FAMILIES OF PERIODIC AND QUASI-PERIODIC ORBITS AROUND L2}

\section{Periodic Orbits}

The linear six-dimensional phase space around L2 is a center $\times$ center $\times$ saddle. For energy values close to that at L2, where it is sufficient to consider only the linear approximation to the equations of motion, there exist two families of periodic orbits; the Horizonal Lyapunov orbits, which are in the ecliptic plane, and the horizontally symmetric figure-eight-shaped Vertical Lyapunov orbits. As the energy is increased, and nonlinear terms become important, the linear phase space is broken and a new periodic family, Halo orbits, bifurcate from the Horizontal Lyapunov orbit family. These orbits are three-dimensional and asymmetric about the ecliptic plane. Figure 1 shows the three distinct periodic orbit families around L2.

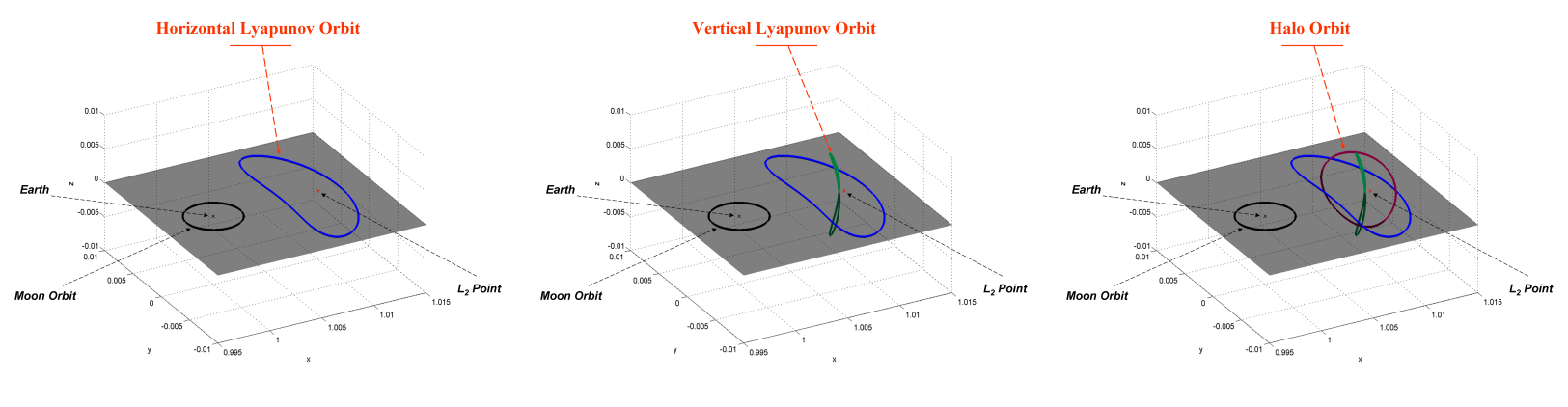

FIGURE 1. Periodic orbits around L2 libration point

\section{Quasi-Periodic Orbits}

The four-dimensional center manifold around L2 is occupied by quasi-periodic orbits of two different families: The Lissajous family around the Vertical Lyapunov orbits, and the Quasi-Halos around the Halo orbits. These quasiperiodic orbits reside on invariant tori about the corresponding periodic orbit. To visualize this four-dimensional center manifold, which consists of all the periodic and quasi-periodic orbits, on a two-dimensional figure, we need to constrain the center manifold by two dimensions. A convenient way of achieving this is to choose periodic and quasi-periodic orbits which have the same energy, and to take a Poincare section when these orbits cross the ecliptic plane. When we do this we obtain Figure 3. Since this is a Poincaré section, the equilibrium points correspond to the periodic orbits of the original system, while the closed curves correspond to the quasi-periodic orbits. This correspondence is shown in Figure 3. Detailed approaches for obtaining these orbits are discussed in the following sections. 

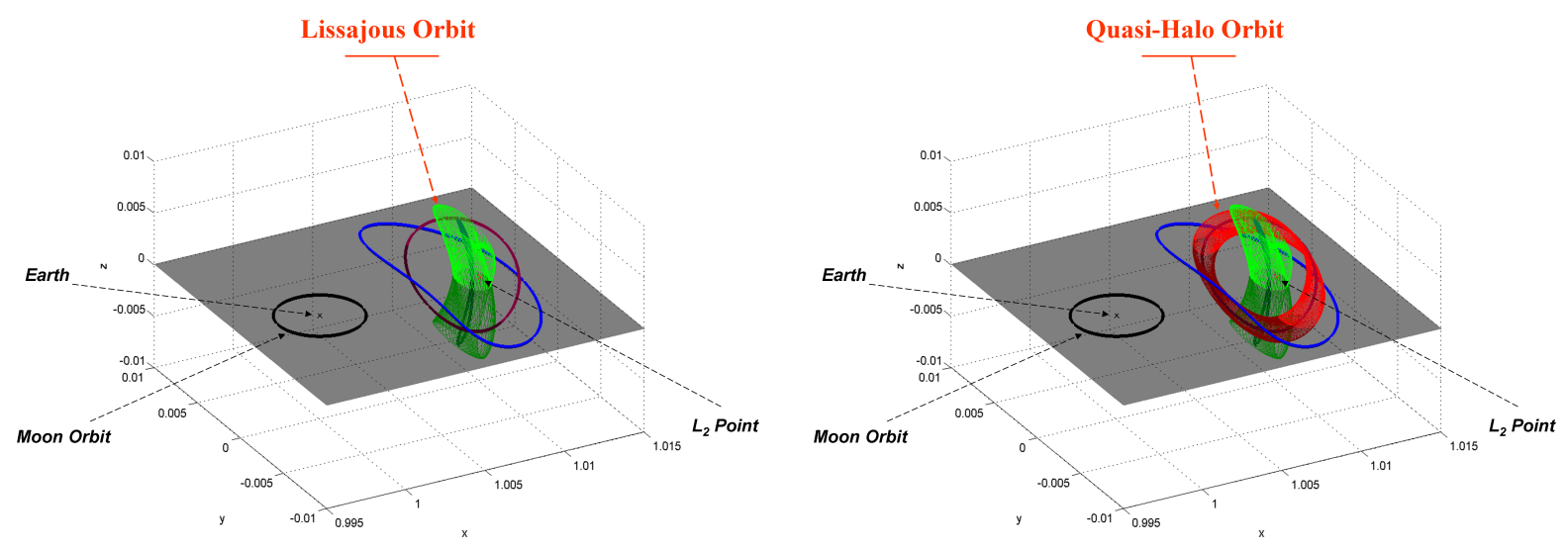

FIGURE 2. Quasi-periodic orbits around L2

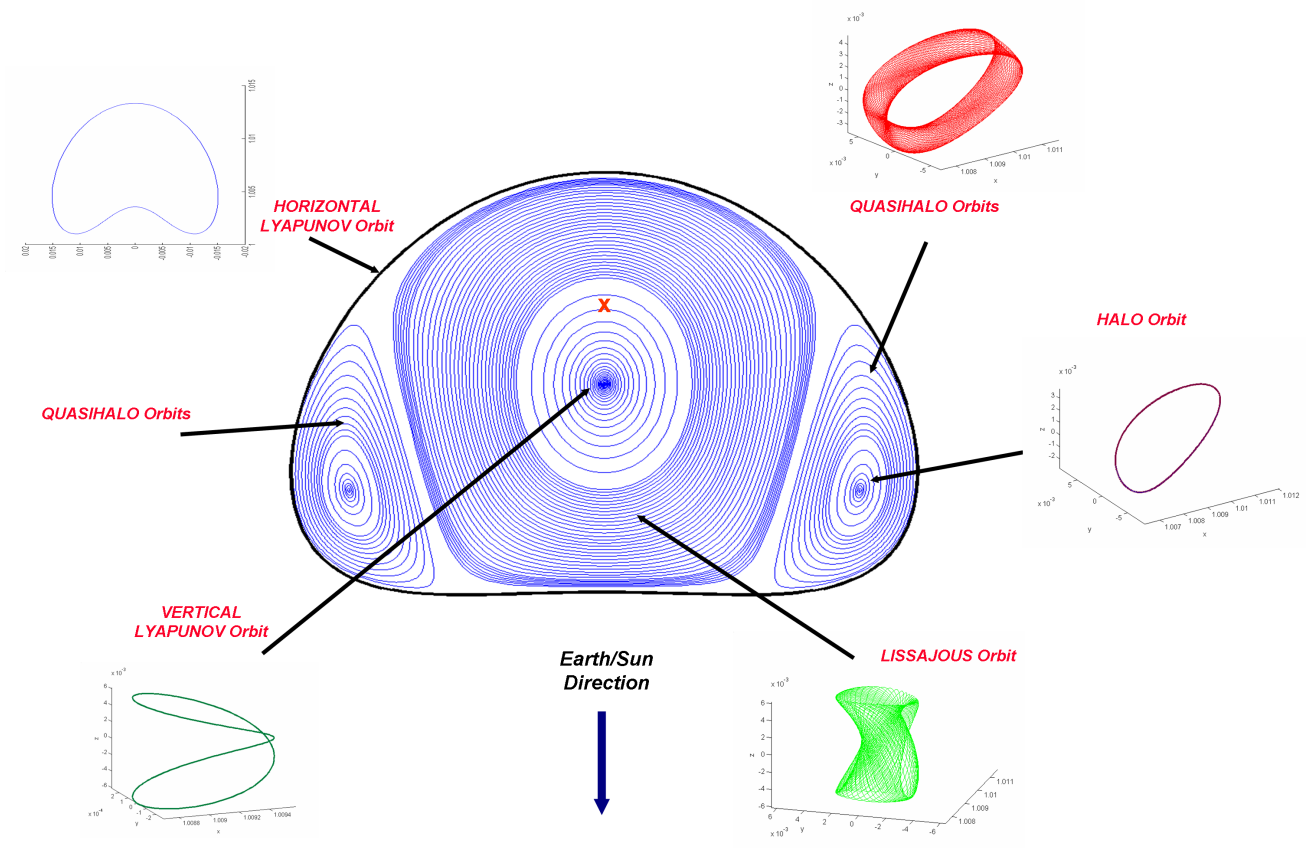

FIGURE 3. All the periodic and quasi-periodic orbits around L2 shown on a Poincaré section of the ecliptic plane

\section{FINDING QUASI-PERIODIC ORBITS VIA MULTIPLE POINCARÉ SECTIONS}

We identify three main challenges associated with finding quasi-periodic orbits. The first is to minimize the time it takes to program the software that numerically solves for the orbits. This reduces the threshold for researchers who work on trajectory design. The second is to achieve reasonable execution times, allowing results to be obtained quickly and "on the go". The third is to improve the robustness of the method in order to get the very large regions of attraction that are needed to obtain the full families of quasi-periodic orbits.

Symbolic methods such as the Lindstedt-Poincaré method [13] and the reduction to the center manifold [11, 12], 
which depend on series expansions, are very slow because an exponential increase in the number of coefficients is needed for every additional increase in the order of expansion. The speed problem can be overcome to a great extent by programming a symbolic manipulator for the problem of interest, but this leads to a significant increase in programming time. More importantly, some of these techniques have instability problems near resonances.

This led us to consider fully numeric methods. However, instead of taking a mesh on the whole surface, which requires many points and is thus memory and CPU intensive, we considered only a section on the torus containing the quasi-periodic trajectories and thus represented the full torus by only points on this section. These points must be integrated for one period at each iteration step. The initial errors in our estimation of the section increase exponentially with the highest Lyapunov exponent as the integration time increases. This is of great concern, especially for the RTBP L2 case where the Lyapunov exponent is more than $10^{3}$. The effect is more dramatic near resonance and chaotic regions. To overcome this problem, we reduce the integration times by taking multiple sections on the torus, integrating only between the consecutive Poincaré sections. The methodology is parallel to the multiple shooting method used in two-point boundary value problems [16].

In what follows, we first introduce the methodology by explaining the procedure for a single Poincaré section. The results are then extended to multiple Poincaré sections. We discuss different implementations using Poincaré sections on phase space and time. Finally, a continuation method to find the full family of quasi-periodic orbits is introduced.

\section{Finding Invariant Tori via a Single Poincaré Section}

The first step in the procedure is to find a convenient Poincaré section. This can be either a section in time or space. For visualization purposes, we assume that the section is taken in phase space, as shown in Figure 4 . When choosing the plane of the section, the main concern is to ensure that the velocity vector of the quasi-periodic orbit is as transverse to the plane of section as possible. This reduces the possibility that the integrated points will not return to the Poincaré section. Thus, a good candidate for the Poincaré section is the plane perpendicular to the velocity of the halo orbit section. However, for the RTBP, a simple section on the ecliptic plane is also suitable. We used both types of Poincaré sections for the results in this paper.

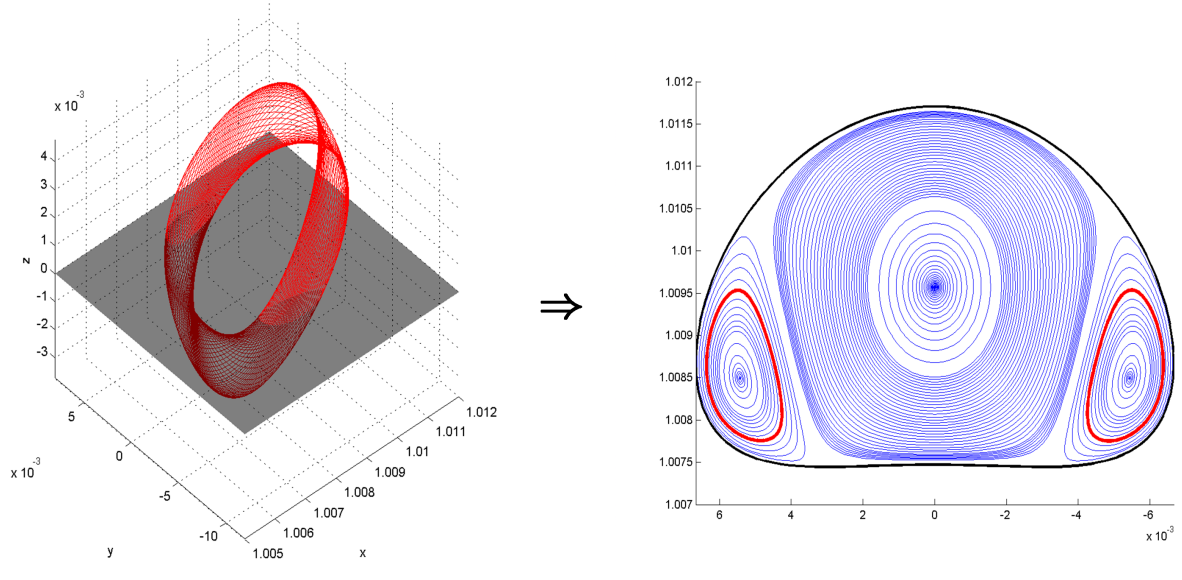

FIGURE 4. Converting the search for a torus problem of a differential equation to the search of the circle of a map

Instead of trying to find the torus of the full ordinary differential equation, our next aim is to find the closed orbits of the Poincaré map. We expand the closed orbit in a truncated Fourier series with the expansion parameter $\theta$, the angle on the closed orbit. For instance, in the case where we took the Poincare section on the ecliptic plane, i.e. $z=0$, the expansion parameter becomes $\theta=\operatorname{atan}\left(\frac{y-y_{\text {halo }}}{x-x_{\text {halo }}}\right)$. It is important to note that we need to parameterize the system such that every point on the closed orbit is uniquely defined by one value of the parameter. For closed orbits with complex shapes, other parameters, such as the ratio of the arc length between a specific point on the orbit and the full arc length of the closed orbit [17], should be used to ensure uniqueness. For the RTBP, however, this simple parameter gives satisfactory results.

We then take sample points, $\mathbf{X}_{\mathbf{0}}$, on the closed orbit:

$$
\mathbf{X}_{\mathbf{0}}=\sum_{n=-n_{m}}^{n_{m}} Q_{n} e^{i n \theta} .
$$


We map these points, $\mathbf{P}\left(\mathbf{X}_{\mathbf{0}}\right)$, by integrating the equations of motion until they intersect the Poincaré section,

$$
\mathbf{X}_{\mathbf{T}}=\mathbf{P}\left(\mathbf{X}_{\mathbf{0}}\right)=\phi_{T\left(\mathbf{X}_{\mathbf{0}}\right)}\left(\mathbf{X}_{\mathbf{0}}\right) ;
$$

The first variations of these equations are integrated along with $\mathbf{X}_{\mathbf{0}}$, for later use in the iteration process. As seen in Figure 5, finding the quasi-periodic orbit is then reduced to solving for the Fourier coefficient vector $\mathbf{Q}$ that satisfies the following equation,

$$
F(\mathbf{Q})=\mathbf{X}_{\mathbf{T}}-\sum_{n} Q_{n} e^{i n \theta\left(\mathbf{X}_{\mathbf{T}}\right)}=0 .
$$

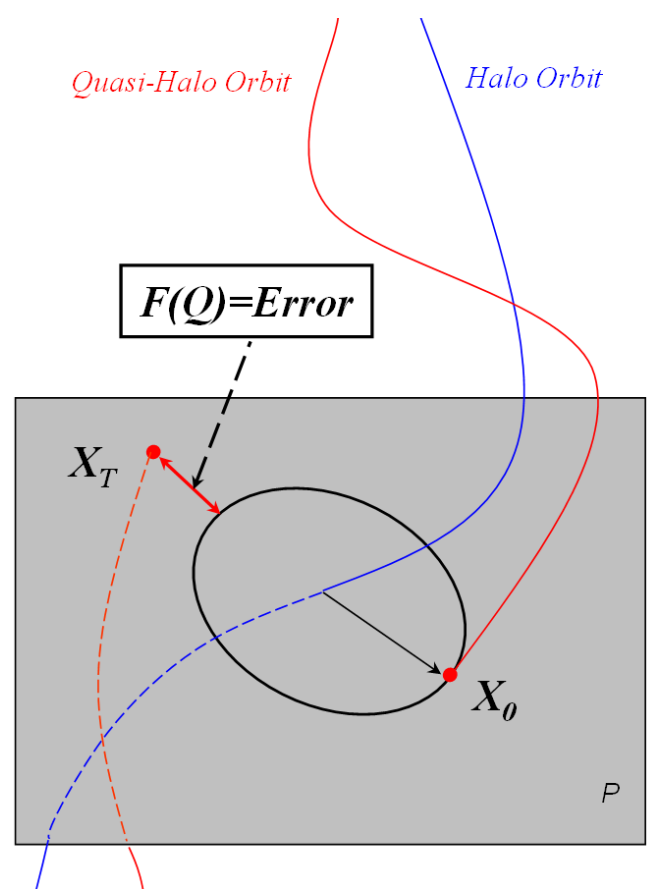

FIGURE 5. Schematic illustration of the numerical procedure for finding the invariant torus

One advantage of this formulation is that we can use a Newton iteration and get quadratically convergent solutions. In Newton's iteration, an initial guess $\mathbf{Q}^{\mathbf{1}}$ is iterated according to

$$
D F\left(\mathbf{Q}^{\mathbf{j}}\right) \cdot\left(\mathbf{Q}^{\mathbf{j}+\mathbf{1}}-\mathbf{Q}^{\mathbf{j}}\right)=-F\left(\mathbf{Q}^{\mathbf{j}}\right)
$$

until a satisfactory answer is reached. To find an explicit form for this equation, we write $\mathbf{X}$ as a matrix multiplication,

$$
\mathbf{X}=\sum_{n} Q_{n} e^{i n \theta}=M(\theta) \cdot \mathbf{Q}
$$

$\mathrm{F}$ then becomes,

$$
\begin{aligned}
F(\mathbf{Q}) & =\mathbf{X}_{\mathbf{T}}-\mathbf{X}_{\theta_{\phi\left(\mathbf{x}_{\mathbf{0}}\right)}} \\
& =\phi(M \cdot \mathbf{Q})-M_{\theta_{\phi(M \mathbf{Q})}} \cdot \mathbf{Q}
\end{aligned}
$$

We then take the derivative with respect to $\mathbf{Q}$,

$$
\begin{aligned}
D F(\mathbf{Q}) & =\frac{d X_{T}}{d X_{0}} \frac{d X_{0}}{d Q}-\frac{d X}{d \theta} \frac{d \theta}{d X_{T}} \frac{d X_{T}}{d X_{0}} \frac{d X_{0}}{d Q} \\
& =D P \cdot M-\left(D M_{\theta_{\phi(M \cdot \mathbf{Q})}} \cdot \mathbf{Q}\right) \cdot \frac{d \theta}{d X_{T}} \cdot D P \cdot M
\end{aligned}
$$

where $D P$ is the differential of the Poincare map obtained from the first variation of the map integrated with $\mathbf{X}$. Employing Newton's iteration, solutions converge usually within 3-4 iterations. An example of an iteration procedure is shown in Figure 6. Here the sample points are shown with red crosses and the return maps are shown by blue circles. After four iterations, all the estimates and the return maps are aligned on the same closed orbit. 

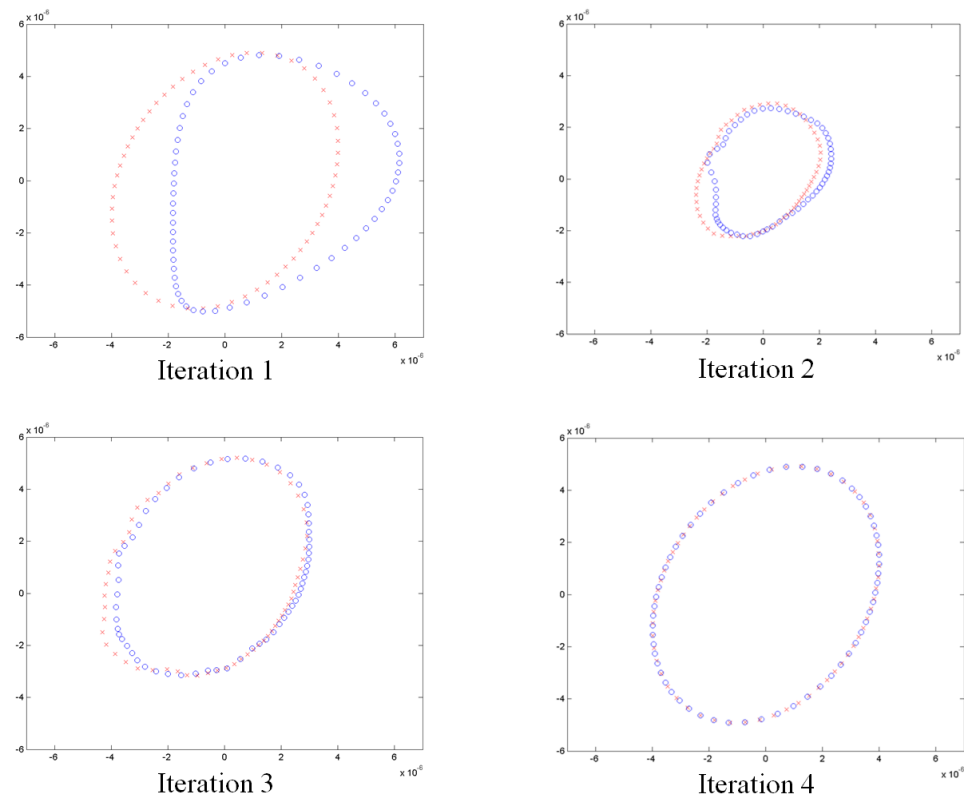

FIGURE 6. Iteration procedure in steps

\section{Extension to Multiple Poincaré Sections}

In order to overcome the potential instability that results from the long integration times, the invariant torus can be cut by several Poincaré sections, as mentioned before. Figure 7 shows the closed orbits which we obtain when the tori of interest are cut with multiple sections. As a result, all of these closed orbits in Figure 7 will be searched instead of only the single one in the previous sub-section.
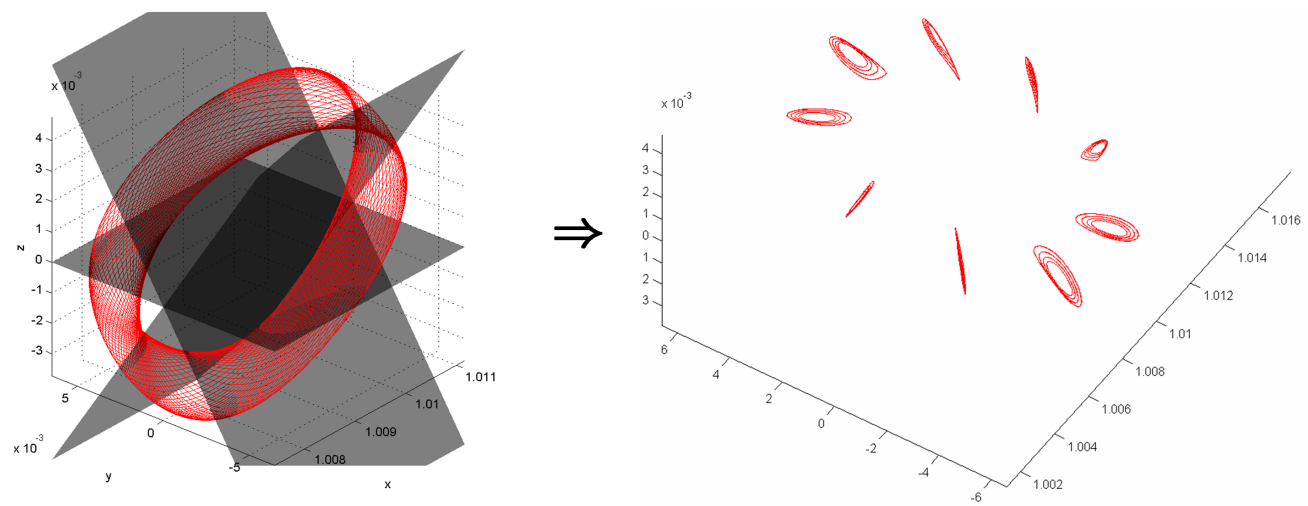

FIGURE 7. Multiple Poincaré Section Procedure: Closed orbits obtained by sectioning invariant tori

The numerical procedure is similar to the single Poincaré section method but in this case, $\mathbf{Q}$, the vector containing the Fourier coefficients, is composed of the Fourier coefficients of each of the closed orbits,

$$
q_{i}=\mathbf{Q}_{n_{i}}^{t}, \quad i=1,2, \ldots, N_{\text {Poincaré sections }} \quad n=1,2, \ldots, N_{\text {Fourier series }}
$$


The invariance condition for these closed orbits to be on the same torus is

$$
F(\mathbf{Q})=F\left(\begin{array}{l}
q_{1}^{t} \\
q_{2}^{t} \\
\vdots \\
q_{N-1}^{t} \\
q_{N}^{t}
\end{array}\right)=\left(\begin{array}{l}
\phi\left(M \cdot q_{1}\right) \\
\phi\left(M \cdot q_{2}\right) \\
\vdots \\
\phi\left(M \cdot q_{N-1}\right) \\
\phi\left(M \cdot q_{N}\right)
\end{array}\right)-\left(\begin{array}{l}
M_{\theta_{\phi\left(M \cdot q_{1}\right)}} \cdot q_{2} \\
M_{\theta_{\phi\left(M \cdot q_{2}\right)}} \cdot q_{3} \\
\vdots \\
M_{\theta_{\phi\left(M \cdot q_{N-1}\right)}} \cdot q_{N} \\
M_{\theta_{\phi\left(M \cdot q_{N}\right)}} \cdot q_{1}
\end{array}\right)=0 .
$$

Finally, as before, we apply Newton's iteration to root finding,

$$
D F\left(\mathbf{Q}^{j}\right) \cdot\left(\mathbf{Q}^{j+1}-\mathbf{Q}^{j}\right)=-F\left(\mathbf{Q}^{j}\right) .
$$

\section{Different Implementations}

Depending on how we choose the Poincaré section, the procedure can be implemented in different ways. If we take a Poincare section in phase space, the four-dimensional center manifold is constrained by two dimensions. The first dimensional constraint is due to the Poincare section, and the second one is due to the fact that the closed orbit we are interested in is one-dimensional. As a result, we are left with two degrees of freedom to specify a unique orbit. Thus, we can specify two constraints, which are properties of the orbit that are of interest. We can, for example, specify the Hamiltonian and the size of the orbit. Since we are working on a Poincare section, size can be specified by the area which is enclosed by the closed orbit. Then, the constraint vector is augmented to include the new constraints,

$$
F=\left[F ; H_{\text {fixed }}-H(\mathbf{Q}) ; \text { Area }_{\text {fixed }}-\text { Area }(\mathbf{Q})\right] .
$$

Another implementation is to take a Poincaré section in time. This way we can specify the period of the quasi-periodic orbit along with the integration direction for the return map. This is especially important for space missions that require all the spacecraft to stay close to one another at all times. In this case, we have one more degree of freedom to specify a unique orbit. We can do this by augmenting the error vector with a new constraint, such as the projected size along one direction,

$$
F=\left[F ; \text { Size }_{\text {fixed }}-\operatorname{Size}(\mathbf{Q}) ; \ldots\right]
$$

\section{Continuation Procedure}

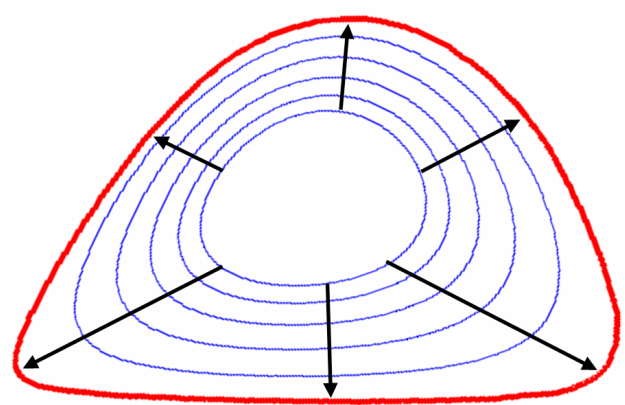

FIGURE 8. Continuation in the area variable

Once we obtain the Fourier coefficients for a given orbit, we would like to extend these results to find the complete family of quasi-periodic orbits. In Figure 8, the continuation in the area variable is shown. While there is no intuitive way to continue the coordinate variables, $[x, y, z, \dot{x}, \dot{y}, \dot{z}]$, the continuation of the Fourier coefficients is straight-forward, since they vary incrementally between sufficiently close quasi-periodic family members. A low-order polynomial fit is thus sufficient for continuing these parameters. Even a very simple linear continuation in the area variable, $A$, gives satisfactory results,

$$
\mathbf{Q}_{k+1}=\mathbf{Q}_{k}+\left(A_{k+1}-A_{k}\right) \frac{\mathbf{Q}_{k}-\mathbf{Q}_{k-1}}{A_{k}-A_{k-1}}
$$




\section{COMPARISON OF THE RESULTS}

The Poincaré section in time, where we specified the orbit period, is shown in Figure 9. Here we compare the results to Gómez et al.'s [13]. By specifying the period of all the orbits to be the same as the base halo orbit's, we can ensure that the spacecraft stay close at all times. This is of great importance particularly for natural, control-free, formation-flying missions. The main advantage of the Poincaré section approach is that, due to the numerical nature of the algorithm, the calculations take only a few minutes of computation time, with minimal programming requirement. Note that, since the Hamiltonians for these orbits are not equal to one another, the orbits may intersect.
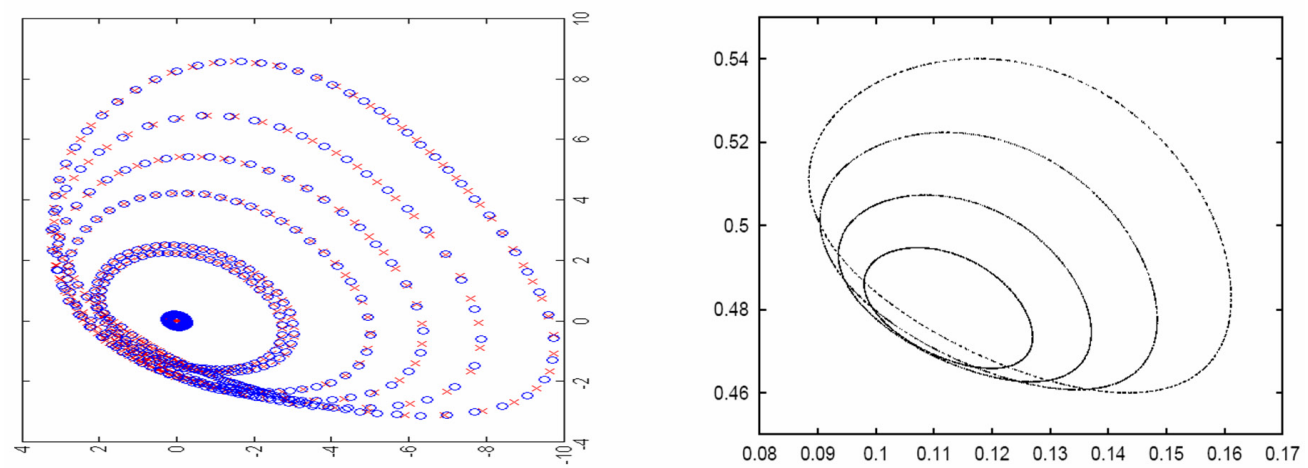

FIGURE 9. The Poincaré section of the invariant tori, where the period of all the orbits is equal to that of the base halo orbit. The multiple Poincaré section method is employed on the left while the Lindstedt-Poincaré method is used on the right [13].

Utilizing the multiple sections approach, we found the complete Quasi-Periodic Orbit Families around the libration points. Figure 10 shows the Poincare section of the quasi-periodic family with constant energy on the ecliptic plane, compares the results obtained by our multiple Poincaré method with Gómez \& Mondelo's refined Fourier analysis $[14,15]$ and Gómez et al.'s Lindstedt-Poincaré analysis [13]. While it was not possible to obtaine the complete families at [13] and a cluster of parallel computers was required to get the complete families at [14, 15], our method obtains the complete picture and has a computation time of only a few minutes on a laptop computer.
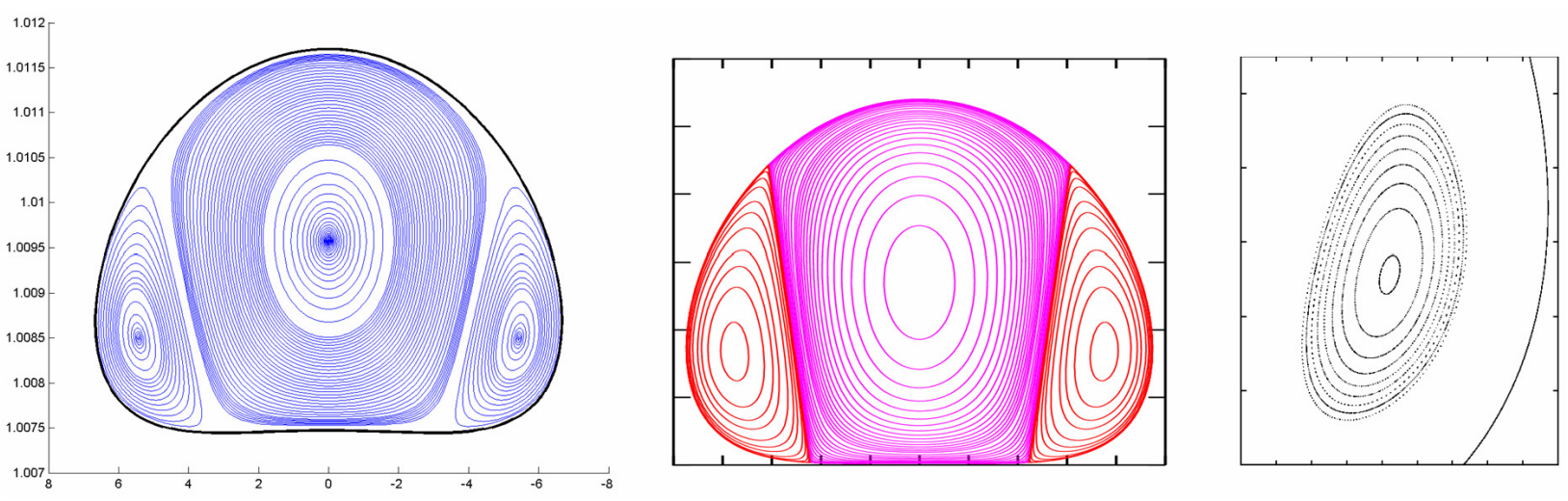

FIGURE 10. The Poincare section of the complete quasi-periodic family around L2 with energy that is equivalent to the 500,000 $\mathrm{km}$-sized halo orbit on the ecliptic plane (on the left). Similar results from [14, 15] (in the middle) and [13] (on the left).

\section{CONCLUSIONS}

This paper shows a fast, stable, easy-to-implement, and highly accurate multiple Poincaré sections method to find quasi-periodic orbits. This method enables specification of the period, size and energy of the quasi-periodic orbit. A continuation method to expand the results from a single solution to a family of orbits is introduced. Quasi-Halo and Lissajous families around the L2 libration point are obtained via this method. The results compare favorably with the existing literature. 


\section{REFERENCES}

1. C. D. Murray, and S. F. Dermott, Solar System Dynamics, Cambridge University Press, 2000.

2. R. Farquhar, Journal of Spacecraft and Rockets 4, 1383-1384 (1967).

3. R. Farquhar, and A. Kamel, Celestial Mechanics 7, 458-473 (1973).

4. M. Hénon, Astronomy \& Astrophysics 28, 415-426 (1973).

5. J. Breakwell, and J. Brown, Celestial Mechanics 20, 389-404 (1979).

6. K. Howell, Celestial Mechanics 32, 53-72 (1984).

7. F. Schilder, H. M. Osinga, and W. Vogt, SIAM J. Applied Dynamical Systems 4, 459-488 (2005).

8. K. Howell, and H. Pernicka, Celestial Mechanics 41, 107-124 (1988).

9. B. T. Barden, and K. C. Howell, Formation Flying in the Vicinity of Libration Point Orbits, AAS Paper No. 98-169, AAS/AIAA Conference, Monterey, California (1998).

10. B. T. Barden, and K. C. Howell, Dynamical Issues Associted with Relative Configurations of Multiple Spacecraft Near the Sun-Earth/Moon L1 Point, AAS Paper No. 99-450, AAS/AIAA Conference, Girdwood, Alaska (1999).

11. A. Jorba, and J. Villanueva, Physica D 114, 197-229 (1998).

12. A. Jorba, and J. Masdemont, Physica D 132, 189-213 (1999).

13. G. Gómez, J. Masdemont, and C. Simó, Journal of The Astronautical Sciences 46, 1-42 (1999).

14. G. Gómez, and J. M. Mondelo, Physica D 157, 283-321 (2001).

15. J. M. Mondelo, Contribution to the Study of Fourier Methods for Quasi-Periodic Functions and the Vicinity of the Collinear Libration Points, Ph.D. thesis, Universitat de Barcelona, Departament de Matemàtica Aplicada i Anàlisi (2001).

16. R. M. M. Mattheij, and G. W. M. Staarink, SIAM J. Sci. Stat. Comp. 5, 87-14 (1984).

17. I. G. Kevrekidis, R. Aris, L. D. Schmidt, and S. Pelikan, Physica D 16, 243-251 (1985). 